lar dystrophy, where replacement of specific gene products such as the sarcoglycans could easily be detected. Mesoangioblasts, on the other hand, have been shown to be efficacious in restoring expression of $\alpha$-sarcoglycan in the $\alpha$-sarcoglycan-null mouse as well as the expression of the whole dystrophin complex, including $\delta$-sarcoglycan (20). All of these cell types are easy to expand in culture and, once their homing and muscle differentiation activities can be optimized, they may represent a better perspective for the stem cell therapy of striated muscle diseases than BM-SP stem cells.

\section{Acknowledgments}

I would like to thank G.L. Condorelli and M. Sampaolesi for critical reading of the manuscript.

Address correspondence to: Giulio Cossu, Stem Cell Research Institute, DIBIT, H. San Raffaele, 58 Via Olgettina, 20132 Milan, Italy. Phone: 3902-2156-0250; Fax: 39022156-0220; E-mail: cossu.giulio@hsr.it.
1. Goodell, M.A., et al. 1996. Isolation and functional properties of murine hematopoietic stem cells that are replicating in vivo. J. Exp. Med. 183:1797-1806.

2. Gussoni, E., et al. 1999. Dystrophin expression in the mdx mouse restored by stem cell transplantation. Nature. 401:390-394

3. Orlic, D., et al. 2001. Bone marrow cells regenerate infarcted myocardium. Nature. 410:701-705.

4. Coral-Vazquez, R., et al. 1999. Disruption of the sarcoglycan-sarcospan complex in vascular smooth muscle: a novel mechanism for cardiomyopathy and muscular dystrophy. Cell. 98:465-474.

5. Seale, P., and Rudnicki, M.A. 2000. A new look at the origin, function, and "stemcell" status of muscle satellite cells. Dev. Biol. 218:115-124.

6. Beltrami, A.P., et al. 2003. Adult cardiac stem cells are multipotent and support myocardial regeneration. Cell. 114:763-776.

7. Oh, H., et al. 2003. Cardiac progenitor cells from adult myocardium: homing, differentiation, and fusion after infarction. Proc. Natl. Acad. Sci. U. S. A. 100:12313-12318.

8. Lapidos, K.A., et al. 2004. Transplanted hematopoietic stem cells demonstrate impaired sarcoglycan expression after engraftment into cardiac and skeletal muscle J. Clin. Invest. 114:1577-1585. doi:10.1172/JCI200423071.

9. Lu, Q.L., et al. 2000. Massive idiosyncratic exon skipping corrects the nonsense mutation in dystrophic mouse muscle and produces functional revertant fibers by clonal expansion. J. Cell Biol. 148:985-996.

10. Ferrari, G., et al. 1998. Skeletal muscle regeneration by bone marrow derived myogenic progenitors. Science. 279:1528-1530.

11. Kelly, R., et al. 1995. Myosin light chain 3F regulatory sequences confer regionalised cardiac and skeletal muscle expression in transgenic mice. J. Cell Biol. 129:383-396.

12. Arnold, H.H., and Winter, B. 1998 Muscle differentiation: more complexity to the network of myogenic regulators. Curr. Opin. Genet. Dev. 8:539-544.

13. Weiss, A., and Leinwand, L.A.1996. The mammalian myosin heavy chain gene family. Annu. Rev. Cell Dev. Biol. 12:417-439.

14. Liu, L.A., and Engvall, E. 1999. Sarcoglycan isoforms in skeletal muscle. J. Biol. Chem. 274:38171-38176.

15. Cossu, G., and Sampaolesi, M. 2004. New therapies for muscular dystrophy: cautious optimism. Trends Mol. Med. 10:516-520

16. Itescu, S., Schuster, M.D., and Kocher, A.A. 2003. New directions in strategies using cell therapy for heart disease. J. Mol. Med. 81:288-296.

17. Liechty, K.W., et al. 2000. Human mesenchymal stem cells engraft and demonstrate site specific differentiation after in utero transplantation in sheep. Nat. Med. 6:1282-1286.

18. Reyes, M., et al. 2001. Purification and ex vivo expansion of postnatal human marrow mesodermal progenitor cells. Blood. 98:2615-2625.

19. Galli, R., et al. 2000. Skeletal myogenic potential of adult neural stem cells. Nat. Neurosci. 3:986-991.

20. Sampaolesi, M., et al. 2003. Cell therapy of alpha sarcoglycan null dystrophic mice through intraarterial delivery of mesoangioblasts. Science. 301:487-492.

\title{
Marfan syndrome and mitral valve prolapse
}

\author{
Arthur E. Weyman and Marielle Scherrer-Crosbie
}

Cardiac Ultrasound Laboratory, Cardiology Division, Department of Medicine, Massachusetts General Hospital, Boston, Massachusetts, USA.

\begin{abstract}
Mitral valve prolapse (MVP), an abnormal displacement into the left atrium of a thickened and redundant mitral valve during systole, is a relatively frequent abnormality in humans and may be associated with serious complications. A recent study implicates fibrillin-1, a component of extracellular matrix microfibrils, in the pathogenesis of a murine model of MVP (see the related article beginning on page 1586). This investigation represents an initial step toward understanding the mechanisms involved in human MVP disease and the development of potential treatments.
\end{abstract}

\section{Mitral valve prolapse: scope of the problem and evolution of the defining criteria}

Mitral valve prolapse (MVP) is generally understood to be the displacement of abnormally thickened, redundant mitral leaflet(s) into the left atrium during systole (Figure 1) (1). One of the possible consequences of this condition is that the malfunctioning mitral valve allows back-

Nonstandard abbreviations used: FBN1, fibrillin-1; MVP, mitral valve prolapse; TGFBR2, TGF- $\beta$ receptor 2 .

Conflict of interest: The authors have declared that no conflict of interest exists.

Citation for this article: J. Clin. Invest. 114:1543-1546 (2004). doi:10.1172/JCI200423701. flow of blood in the left atrium (mitral regurgitation), which, when severe, leads to left ventricular enlargement and failure. Besides severe mitral regurgitation, MVP has been associated with serious complications such as bacterial endocarditis and sudden death, and primary mitral valve surgery is currently performed most frequently to specifically treat $\operatorname{MVP}(1,2)$. Since the early 1970s, research has suggested that echocardiography is the ideal noninvasive technique to visualize the prolapsing mitral leaflets (3-5). However, continually changing echocardiographic techniques and criteria for the diagnosis of MVP have in many cases further obscured, rather than clarified, our understanding of prolapse in its primary form and in association with other disorders (6). Over the past decade, new echocardiographic criteria for MVP have been established based on an understanding of the 3D structure of the mitral valve (7). Defined according to these criteria, prolapse is the displacement of 1 or both mitral leaflets by more than $2 \mathrm{~mm}$ above the high points of the mitral annulus as recorded in either the parasternal or apical long-axis view (Figure 1 , detail). This $2 \mathrm{~mm}$ displacement derives from studies showing that smaller displacements are not associated with increased leaflet thickness, mitral regurgitation, or valve-related complications (8). In cases where leaflet displacement is greater than $2 \mathrm{~mm}$, prolapse is further subdivided into classic and nonclassic forms based on leaflet thickness (classic, $>5 \mathrm{~mm}$; and nonclassic, $\leq 5 \mathrm{~mm}$ ), with complications such as endocarditis or severe mitral regurgitation generally occurring in patients with classic prolapse (9).

Using these criteria, a recent population study of 3,491 subjects from the offspring 
cohort of the Framingham Heart Study reported a prevalence of $1.3 \%$ for classic MVP and 1.1\% for the nonclassic form (10). This overall prevalence of $2.4 \%$ is strikingly lower than the extremely high values previously reported elsewhere (21\% and 34\% when diagnosed using M-mode diagnostic ultrasound or 2D electrocardiographic views, respectively) and significantly lower than the more generally quoted figures of $4-7 \%$ for the general population based on older M-mode studies $(1,11)$. In the recent study of the offspring cohort of the Framingham Heart Study (10), the incidence of MVP did not differ between the sexes, and no predisposition to prolapse was observed in younger or older adult subjects; this was supported by an even distribution (2-3\%) among subjects in each decade from 30 to 80 years. Other data regarding the prevalence of MVP in children suggest that prolapse is uncommon before adolescence, but its prevalence increases after the adolescent growth spurt $(12,13)$. Nonclassic prolapse appears morphologically to be an intermediate stage between normal mitral valve anatomy and classic prolapse, but studies with up to a 10-year follow-up have shown no evidence of progression from one form to another, and the Framingham Heart Study failed to show an age-related shift from one type to the other, although the patient numbers studied in each age group were small.

\section{Mitral valve prolapse and mitral regurgitation}

The most common complication of MVP is severe regurgitation due to progressive degeneration of the valve and chordae, with myxomatous infiltration (thickening of the mitral layers with glycosaminoglycan accumulation), and fibroelastic and collagen alterations $(10,11,13)$. In about $75 \%$ of cases, there is sudden deterioration because of chordal rupture (14). The cumulative risk of severe mitral regurgitation and valve rupture is minimal in individuals younger than 50 years of age but then rises steeply, with the risk in men being greater than in women beyond the age of 60 . Using current prevalence data, it can be estimated that by the age of 70 , approximately $11 \%$ of men and $6 \%$ of women with classic MVP will need mitral valve replacement (13).

\section{Mitral valve prolapse: a genetic disease?}

MVP is generally sporadic but is also associated with a variety of congenital disor- ders of connective tissue including Marfan syndrome, Ehler-Danlos syndrome, osteogenesis imperfecta, dominant cutis laxa pseudoxanthoma elasticum, and the MASS syndrome (mitral valve prolapse, a dilatation, skeletal changes, and skin changes), among others. It has been estimated that only $0.25 \%$ of patients with MVP have Marfan syndrome. This percentage may be somewhat higher if the newer and more stringent criteria for MVP are used, but it is unlikely that more than $1-2 \%$ of patients with MVP have an associated connective tissue disorder. Marfan syndrome is associated with mutations in Fibrillin-1 (FBN1) on chromosome 15q21.1 $(15,16)$. Because MVP is found in many, but certainly not all, patients with Marfan syndrome, it was suggested many years ago that isolated MVP may also be due to a mutation of FBN1 (15). However, despite the availability of literally millions of patients for study, no convincing association has been found to date. Occasional families with MVP have been identified and an underlying gene defect reported. In patients with X-linked myxomatous valvular dystrophy, a rare disorder associated with severe MVP, the defect has been linked to chromosome Xq28 (17). The first locus for nonsyndromic MVP has been mapped to chromosome 16p11.2p12.2 in 2 of 4 patients at a French surgical center (18). A second locus for autosomal dominant MVP has been mapped to chromosome 11p15.4 (19). Thus, even within families with an autosomal dominant mode of inheritance, there appears to be significant genetic heterogeneity.

The role of mechanical stress in the evolution of MVP is also important. The mitral valve opens and closes more than 3 billion times during the course of the normal human lifespan. During each closure period, it must withstand the full force of ventricular contraction. Because of the normal orientation of the leaflets, this force is unevenly applied, affecting the posterior leaflet more than the anterior leaflet, and prolapse of the posterior leaflet is more common (observed in two-thirds of cases, with prolapse of both leaflets occurring in the remaining third). This pattern of involvement and the fact that the valve disruption occurs late in life are consistent with the important role of physiologic stress in the development of MVP.

\section{Marfan syndrome and the fibrillin-1/TGF- $\beta$ pathway}

Although abnormalities within FBN1 are responsible for the Marfan pheno- type in approximately $80 \%$ of patients, Marfan syndrome can also be caused by inactivation mutations in TGF- $\beta$ receptor 2 (TGFBR2), located at 3p24.2-p25 (20). Fibrillin-1 has also been shown to have a biologically important role in the activation of TGF- $\beta$ in the lung (21). The TGFs are multipotent cytokines that are important modulators of cell growth, proliferation and differentiation, inflammation, extracellular matrix deposition, and apoptosis (22). Virtually every cell in the body produces TGF- $\beta$ and has receptors for it (23). Their biologic effects are context dependant and vary with tissue type and the activity of other signaling pathways (24). Defects in TGF- $\beta$ function are associated with a number of pathologic states, including tumor cell growth, fibrosis, and autoimmune disease (23). TGF- $\beta$ activity is regulated by the conversion of latent TGF- $\beta$ to active TGF- $\beta$. Tissues contain significant quantities of latent TGF- $\beta$, and activation of only a small fraction causes a maximal cellular response. There are multiple activators of latent TGF- $\beta$ (proteases, thrombospondin-1, integrins, reactive oxygen species, and changes in $\mathrm{pH}$ ), and latent TGF- $\beta$ has been considered a molecular sensor that responds to specific signals by releasing active TGF- $\beta$, which is the effector molecule $(22,25)$.

In a study reported in this issue of the JCI, $\mathrm{Ng}$ et al. tested the hypothesis that the fibrillin-1-TGF- $\beta$ pathway is implicated in the pathogenesis of MVP in a murine model of Marfan syndrome (26). They compared the morphometry of mitral valves in fibrilin-1-deficient mice to those of wild-type mice and report a progressive increase in leaflet length and thickness in heterozygous versus homozygous mice. The leaflets exhibited both increased cell proliferation and decreased apoptosis. Using TGF- $\beta$-specific antibodies, they observed that TGF- $\beta$ activity and signaling were increased in the valve tissue, while the concentration of latent TGF- $\beta$ remained unchanged. Most interestingly, the mitral valve phenotype was rescued by TGF- $\beta$-neutralizing antibodies, which confirms a causal relationship between TGF- $\beta$ dysregulation and this abnormal mitral valve.

These findings are consistent with those from other studies that link TGF- $\beta$ to the Marfan phenotype (27) and the demonstration that Marfan syndrome can be caused by inactivation mutations in TGFBR2 at 3 p24.2-p25 (20) in patients without abnor- 


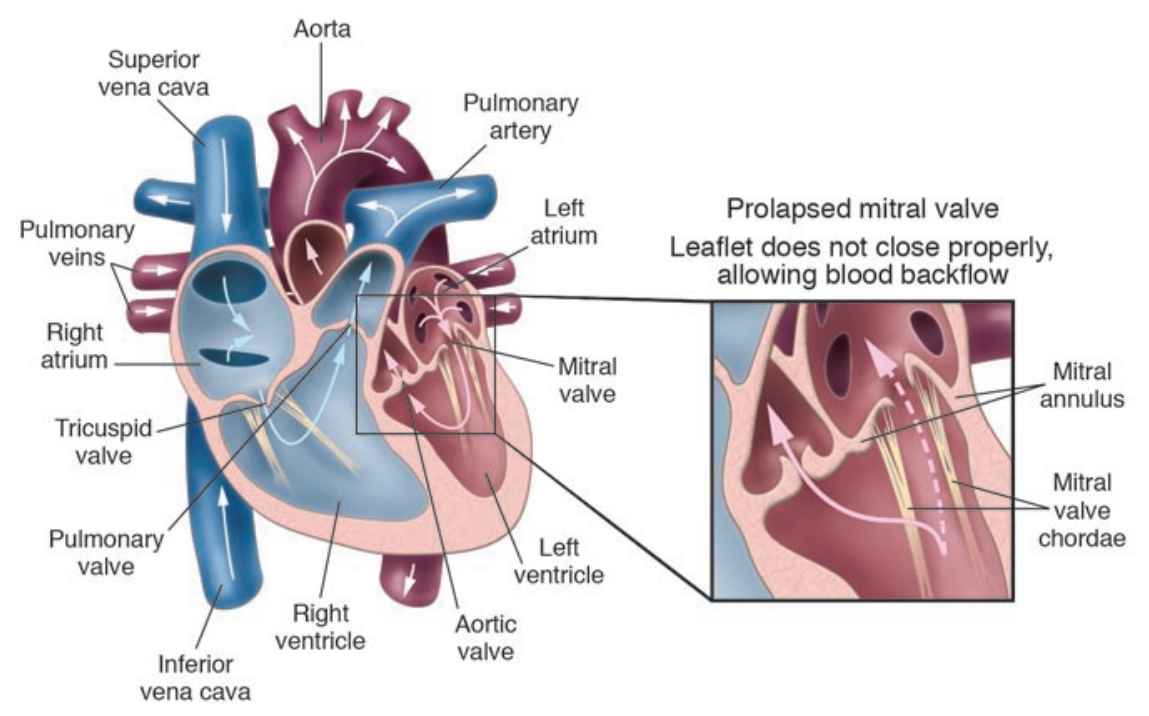

Figure 1

MVP. Mitral valve leaflets, shaped like parachutes, are attached to the inner wall of the left ventricle by a series of strings called chordae. When the ventricles contract, the mitral valve leaflets close snugly to prevent backflow of blood from the left ventricle into the left atrium. Upon ventricle relaxation, the valves open to allow oxygenated blood from the lungs to fill the left ventricle. In patients with MVP, myxomatous degeneration of the leaflets and chordae results in leaflet thickening and redundancy, causing the leaflets to prolapse, or flop backwards, into the left atrium (detail). This sometimes allows leakage of blood through the mitral valve (mitral regurgitation). Severe mitral regurgitation can lead to heart failure and abnormal heart rhythms.

malities in the FBN1 gene. These 2 lines of evidence suggest that abnormalities in the TGF- $\beta$ signaling pathway may represent a final common pathway for the development of the Marfan phenotype.

\section{Murine and human MVP: similarities and differences}

Similar to human MVP, the murine model pioneered by $\mathrm{Ng}$ et al. (26) displays a thickened and redundant mitral valve that prolapses into the left atrium. There are differences, however, among what has been noted in human acquired myxomatous mitral valve disease. Clinically, the MVP that often accompanies Marfan syndrome is not generally considered to be a model of the isolated MVP in the adult for a number of reasons. First, abnormalities in FBN1 have not been demonstrated in sporadic MVP patients or families with the disorder. Second, severe mitral valve disorders in Marfan syndrome are generally manifest in infants, while in adults, the majority of complications are due to aortic aneurysm formation and dissection. This is in contrast to the clinical paradigm of MVP, where complications are typically not seen before the fifth decade of life. Third, the valvular involvement in Marfan syndrome and in these mice affects the come from autopsy or have been surgically removed and thus represent advanced disease, while in the $\mathrm{Ng}$ et al. study, the valves were examined at $0.5,2.5,4.5$, and 6.5 postnatal days. It would be interesting to study the histologic features of the valves in older heterozygous mice to see whether they are similar to those found in clinical MVP. The morphological changes of the mitral valve in this murine model were evident shortly after birth, while idiopathic MVP is uncommon in children and generally becomes manifest after the adolescent growth spurt. It may be that clinically undetectable changes are present at birth in MVP and only become apparent later in life, but this remains to be shown. While an increase in TGF- $\beta$ activity may be shown to be present in the idiopathic form of MVP (particularly given the multiplicity of activators, including stress), both the presence and primacy of this relationship have yet to be demonstrated.

Future studies using this model would ideally include a more detailed description of the anatomic features of the murine model. A complete echocardiographic assessment of MVP and of the functional consequences of the valve morphology on the left-atrial and left-ventricular size and left-ventricular function at birth and in older mice would be of particular interest, as would echocardiographic assessment of the effects of TGF- $\beta$-neutralizing antibodies on the aortic dilation present in the model. Since in humans there is considerable phenotypic variability among individual genotypes, it would also be interesting to see how consistent the phenotypic expression is in the murine model. Importantly, while prolapse is noted at 9 months of age in heterozygous mice, it would be useful to show that the treated heterozygous mice do not also go on to develop prolapse due to incomplete protection by the TGF- $\beta$-neutralizing antibodies.

In summary, it appears that MVP is a final common pathway for a variety of genetic and acquired disorders, which presumably weaken connective tissue of the valve and lead to leaflet elongation, thickening, and often degeneration. The finding that TGF- $\beta$ dysregulation in the connective tissue plays an important role in the development of prolapse in Marfan syndrome (26) raises the question as to whether this cytokine may play a role in other forms of prolapse, including sporadic MVP. It has been suggested that the altered extracellular matrix in fibrillin-1-deficient mice cues cells to 
remodel the matrix, and this remodeling is associated with inappropriate and persistent expression of TGF- $\beta$. Localized trauma-related disruption of fibrillin and collagen might also trigger TGF- $\beta$ activation and matrix remodeling. However, a great deal of study remains necessary before the pathogenic mechanisms underlying idiopathic MVP and its relationship to more generalized forms of connective tissue disease are clarified.

Address correspondence to: Arthur E. Weyman, Cardiac Ultrasound Laboratory, Cardiology Division, Department of Medicine, Massachusetts General Hospital, 55 Fruit Street, Boston 02114, Massachusetts, USA. Phone: (617) 724-7738; Fax: (617) 7268383; E-mail: aweyman@partners.org.

1. Devereux, R.B., et al. 1987. Diagnosis and classification of severity of mitral valve prolapse: methodologic, biologic, and prognostic considerations. Am. Heart J. 113:1265-1280.

2. Chavez, A.M., and Cosgrove, D.M. 1988. Surgery for mitral prolapse. Herz. 13:400-404

3. Shah, P.M., and Gramiak, R. 1970. Echocardiographic recognition of mitral valve prolapse [abstract]. Circulation. 42(Suppl. III):III-45.

4. Dillon, J.C., Haine, C.L., Chang, S., and Feigenbaum, H. 1971. Use of echocardiography in patients with prolapsed mitral valve. Circulation. 43:503-507.

5. Kerber, R.E., Isaeff, D.M., and Hancock, E.W. 1971. Echocardiographic patterns in patients with the syndrome of systolic click and late systolic murmur. N. Engl. J. Med. 284:691-693.

6. Levine, R.A., and Weyman, A.E. 1984. Mitral valve prolapse: a disease in search of, or created by, its definition. Echocardiography. 1:3-14.

7. Levine, R.A., Triulzi, M.O., Harrigan, P., and Weyman, A.E. 1987. The relationship of mitral annular shape to the diagnosis of mitral valve prolapse. Circulation. 75:756-767.

8. Levine, R.A., Stathogiannis, E., Newell, J.B., Harrigan, P., and Weyman, A.E. 1988. Reconsideration of echocardiographic standards for mitral valve prolapse: lack of association between leaflet displacement isolated to the apical four chamber view and independent echocardiographic evidence of abnormality. J. Am. Coll. Cardiol. 11:1010-1019.

9. Marks, A.R., Choong, C.Y., Sanfilippo, A.J., Ferre, M., and Weyman, A.E. 1989. Identification of highrisk and low-risk subgroups of patients with mitralvalve prolapse. N. Engl. J. Med. 320:1031-1036.

10. Freed, L.A., et al. 1999. Prevalence and clinical outcome of mitral-valve prolapse. N. Engl. J. Med. 341:1-7.

11. Levy, D., and Savage, D. 1987. Prevalence and clinical features of mitral valve prolapse. Am. Heart J. 113:1281-1290

12. Hickey, A.J., and Wilcken, D.E. 1986. Age and the clinical profile of idiopathic mitral valve prolapse. Br. Heart J. 55:582-586.

13. Wilcken, D.E., and Hickey, A.J. 1988. Lifetime risk for patients with mitral valve prolapse of developing severe valve regurgitation requiring surgery. Circulation. 78:10-14.

14. Jeresaty, R.M. 1985. Mitral valve prolapse and ruptured chordae tendineae. Am. J. Cardiol. 55:138-142.

15. Dietz, H.C., et al. 1991. Marfan syndrome caused by a recurrent de novo missense mutation in the fibrillin gene. Nature. 352:337-339.

16. Robinson, P.N., et al. 2002. Mutations of FBN1 and genotype-phenotype correlations in Marfan syndrome and related fibrillinopathies. Hum. Mut. 20:153-161.

17. Kyndt, F., et al. 1998. Mapping of X-linked myxomatous valvular dystrophy to chromosome Xq28. Am. J. Hum. Genet. 62:627-632.

18. Disse, S., et al. 1999. Mapping of a first locus for autosomal dominant myxomatous mitral-valve prolapse to chromosome $16 \mathrm{p} 11.2$-p12.1. Am. J.
Hum. Genet. 65:1242-1251.

19. Freed, L.A., et al. 2003. A locus for autosomal dominant mitral valve prolapse on chromosome 11p15.4. Am. J. Hum. Genet. 72:1551-1559.

20. Mizuguchi, T., et al. 2004. Heterozygous TGFBR2 mutations in Marfan syndrome. Nat. Genet. 36:855-860.

21. Kaartinen, V., and Warburton, D. 2003. Fibrillin controls TGF $\beta$ activation. Nat. Genet. 33:331-332.

22. Annes, J.P., Munger, J.S., and Rifkin, D.B. 2003. Making sense of latent TGFbeta activation. J. Cell. Sci. 116:217-224.

23. Blobe, G.C., Schiemann, W.P., and Lodish, H.F. 2000. Role of transforming growth factor beta in human disease. N. Engl. J. Med. 342:1350-1358.

24. Akhurst, R.J. 2004. TGF beta signaling in health and disease. Nat. Genet. 36:790-792.

25. Piek, E., Heldin, C.H., and Ten Dijke, P. 1999. Specificity, diversity, and regulation in TGF-beta superfamily signaling. FASEB J. 13:2105-2124.

26 . Ng, C.M., et al. 2004. TGF- $\beta$-dependent pathogenesis of mitral valve prolapse in a mouse model of Marfan syndrome. J. Clin. Invest. 114:1586-1592. doi:10.1172/JCI200422715.

27. Neptune, E.R., et al. 2003. Dysregulation of TGFbeta activation contributes to pathogenesis in Marfan syndrome. Nat. Genet. 33:407-411.

28. Davies, M.J., Moore, B.P., and Braimbridge, M.V. 1978. The floppy mitral valve. Study of incidence, pathology, and complications in surgical, necropsy, and forensic material. Br. Heart J. 40:468-481.

29. Yetman, A.T., Bornemeier, R.A., and McCrindle, B.W. 2003. Long-term outcome in patients with Marfan syndrome: is aortic dissection the only cause of sudden death? J. Am. Coll. Cardiol. 41:329-332.

30. Kartunnen, L., Raghunath, M., Lonnquvist, L., and Peltonen, L. 1994. A compound heterozygous Marfan patient: two defective fibrillin alleles result in a lethal phenotype. Am. J. Hum. Genet. 55:1083-1091.

31. Rabkin, E., et al. 2001. Activated interstitial myofibroblasts express catabolic enzymes and mediate matrix remodeling in myxomatous heart valves. Circulation. 104:2525-2532. 